\title{
Two-Dimensional Rayleigh Model of Vapor Bubble Evolution
}

\author{
M. Strauss, M. Friedman, E. Gurewitz, \\ P. Amendt, R. A. London, M. E. Glinsky
}

This paper was prepared for submittal to the

Photonics West '99 Symposium

San Jose, CA

January 23-29, 1999

January 14, 1999

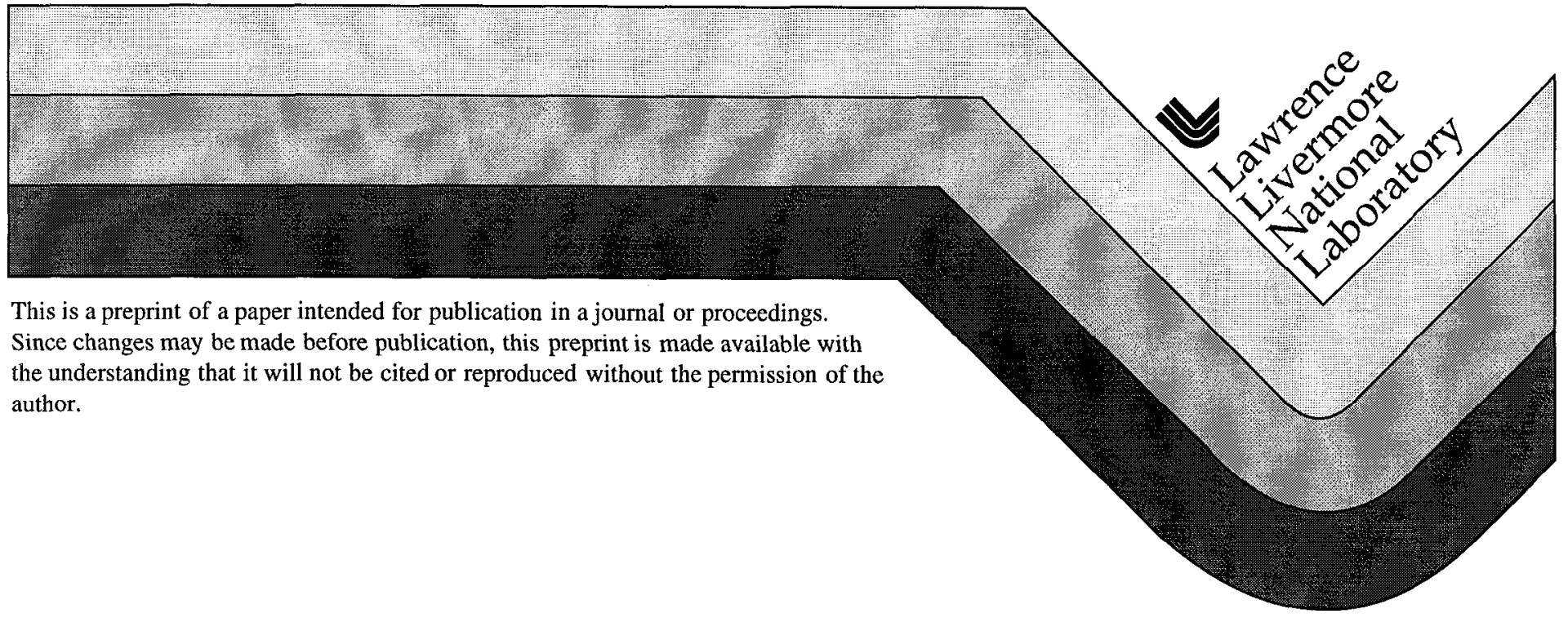


This document was prepared as an account of work sponsored by an agency of the United States Government. Neither the United States Government nor the University of California nor any of their employees, makes any warranty, express or implied, or assumes any legal liability or responsibility for the accuracy, completeness, or usefulness of any information, apparatus, product, or process disclosed, or represents that its use would not infringe privately owned rights. Reference herein to any specific commercial product, process, or service by trade name, trademark, manufacturer, or otherwise, does not necessarily constitute or imply its endorsement, recommendation, or favoring by the United States Government or the University of California. The views and opinions of authors expressed herein do not necessarily state or reflect those of the United States Government or the University of California, and shall not be used for advertising or product endorsement purposes. 


\title{
Two-Dimensional Rayleigh model of vapor bubble evolution
}

\author{
Moshe Strauss, Menahem Friedman, Eitan Gurewitz \\ Peter Amendt", Richard. A. London" and Michael E. Glinsky ${ }^{+}$ \\ Nuclear Research Center, Negev, P.O.Box 9001, Beer Sheva, Israel \\ *Lawrence Livermore National Laboratory, Livermore, CA 94550 \\ + Shell E \& P Technology Co. Houston, TX 77025
}

\begin{abstract}
$\underline{\text { ABSTRACT }}$
The understanding of vapor bubble generation in an aqueous tissue near a fiber tip has required advanced two dimensional (2D) hydrodynamic simulations. For $1 D$ spherical bubble expansion a simplified and useful Rayleigh-type model can be applied. For 2D bubble evolution, such a model does not exist. The present work proposes a Rayleigh-type model for 2D bubble expansion that is faster and simpler than the $2 \mathrm{D}$ hydrodynamic simulations. The model is based on a flow potential representation of the hydrodynamic motion controlled by a Laplace equation and a moving boundary condition. We show that the $1 \mathrm{D}$ Rayleigh equation is a specific case of our model. The Laplace equation is solved for each time step by a finite element solver using a triangulation of the outside bubble region by a fast unstructured mesh generator. Two problems of vapor bubbles generated by short-pulse lasers near a fiber tip are considered: (a) the outside region has no boundaries except the fiber, (b) the fiber and the bubble are confined in a long channel, which simulates a fiber in a vessel wall. Our simulations for problems of type (a) include features of bubble evolution as seen in experiments, including a collapse away from the fiber tip. A different behavior was obtained for problems of type (b) when the channel boundary is close to the fiber. In this case the bubble's expansion and collapse are both extremely slow in the direction normal to this boundary and distortion of the bubble is observed.
\end{abstract}

Keywords: vapor bubble, Rayleigh model, hydrodynamics, potential flow, finite element

\section{INTRODUCTION}

In many medical therapiès short-pulsed lasers in fibers are used on aqueous tissues generating vapor bubbles near the fiber tip. 'The bubble evolution requires two dimensional (2D) hydrodynamic simulations and therefore special computational capabilities. ${ }^{2}$ In $1 \mathrm{D}$ bubble evolution a simplified treatment exists, based on an extended Rayleigh model. ${ }^{3,4}$ This equation is an ordinary differential equation and can be easily solved. For $2 \mathrm{D}$ bubble evolution such a treatment does not exist. In the present paper we derive a 2D Rayleigh type model which can be used in many medical applications. This method is much faster and simpler than the $2 \mathrm{D}$ hydrodynamic simulations.

Our model is based on applying a flow potential $\phi$ related to the fluid velocity outside the bubble $\vec{u}=-\nabla \phi$, where $\phi$ is a solution of a Laplace equation. ${ }^{5}$ We assume as in the Rayleigh model that the inside of the bubble is uniform in pressure and density and derive a moving. boundary condition for $\phi$ at the bubble boundary. For the limiting ID case our model gives the known ID Rayleigh model. For the general case we solve a cylindrical Laplace equation with Dirichlet $(\phi=0)$ and Neumann (nomal component $(\nabla \phi)_{N}=0$ ) boundary conditions based on 
the bubble boundary motion. For every time step an Unstructured Mesh Finite Element Solver (UMFES) is used to update the triangulation of the region outside the bubble. ${ }^{6.7}$

Two cases are solved. In case (a) a fiber deposits instantaneously a short laser pulse in an aqueous system with a free boundary. We obtain solutions, which include the experimental characteristics of the expansion and the collapse of the bubble away from the fiber. In case (b) we consider a fiber and a bubble confined in a channel. This case includes characteristics of a fiber in a vessel. The presence of the channel reduces the fluid flow normal to the channel and causes a distortion in the bubble evolution and especially in the bubble collapse.

The plan of the paper is as follows: Section 2 discusses the physical model. Section 3 presents the numerical procedure. Computational results and discussion are given in Section 4 and concluding remarks are presented in Section 5.

\section{THE PHYSICAL MODEL}

We consider a vapor bubble generated in an aqueous system by a short-pulse laser near a fiber tip. The bubble evolution requires $2 \mathrm{D}$ simulation. ${ }^{2,8} \mathrm{We}$ assume that the bubble is uniform in pressure as in a Rayleigh bubble and that it evolves adiabatically with entropy $\mathrm{S}_{0}$. Applying the bubble equation of state (EOS), any one of the quantities $P$ (pressure) $\rho$ (density), $T$ (temperature), or $\varepsilon$ (energy) together with $S_{0}$ determine the others. For the region outside the bubble the relevant hydrodynamic equations are the continuity equation,

$$
\frac{\partial \rho}{\partial t}+\nabla \cdot(\rho \vec{u})=0
$$

and the momentum equation for the fluid velocity $u$,

$$
\frac{\partial \vec{u}}{\partial t}+\vec{u} \cdot \nabla \vec{u}=-\frac{\nabla p}{\rho}
$$

Outside the bubble we assume a flow potential $\phi$ such that $\vec{u}=-\nabla \phi$ and consequently obtain from Eq.(2) after integrating from $\bar{r}$ to $\infty$,

$$
-\frac{\partial \phi}{\partial t}+\frac{l}{2}(\nabla \phi)^{2}=h(\infty)-h(\vec{r})
$$

where $d h=d p / \rho$ and

$$
h(\infty)-h(\bar{r})=\int_{\bar{r}}^{\infty} \frac{d p}{\rho}
$$

where $h(\bar{r})$ is the enthalpy at $\bar{r}$ and $h(\infty)$ is the ambient enthalpy at large $r$.

For many cases $\rho \approx$ cons tant we may replace in Eq.(4) $h(\infty)-h(\vec{r})$ by $\left(p_{\infty}-p(\vec{r})\right) / \rho$ and get

$$
\frac{d \phi}{d t}=\frac{p-p_{\infty}}{\rho}-\frac{u^{2}}{2},
$$


where $\frac{d}{d t}=\frac{\partial}{\partial t}+\vec{u} \cdot \nabla, p$ is the pressure at $\vec{r}$ and $p_{\infty}$ is the ambient pressure. By enforcing Eq.(5) at the bubble's boundary, we get

$$
\frac{d \Phi}{d t}=\frac{P-p_{\infty}}{\rho}-\frac{U^{2}}{2},
$$

i.e. a relation between the pressure $P$, velocity $U$ and the flow potential $\Phi$ at the boundary. Equation (6) is the boundary condition for the bubble expansion. The velocity $U$ varies along the bubble boundary and so does $\Phi$ by Eq.(6).

Applying Eq.(1) for $\rho \approx$ constant and using $\vec{u}=-\nabla \phi$, we obtain a Laplace equation for the region outside the bubble,

$$
\nabla^{2} \phi=0
$$

The solution of equation (7) with the moving boundary condition, Eq.(6), present the main ingredients of our 2D time dependent bubble model.

We can show that Eqs.(6) and (7) are consistent with the 1D Rayleigh equation. For the $1 \mathrm{D}$ case the Laplace equation solution is $\phi=C / r$. Using $u=-\partial \phi / \partial r$, we get $\phi=R^{2} U / r$. Inserting $\phi$ in Eq.(5) and taking the boundary limit $r=R$ we get,

$$
R \ddot{R}+\frac{3}{2} U^{2}=\frac{1}{\rho}\left(P-p_{\infty}\right),
$$

which is the $1 \mathrm{D}$ Rayleigh equation. ${ }^{4}$

By assuming that $\rho \approx$ constant we ignore the acoustic emission term. Its effect will be studied in future work. The acoustic emission can be approximated for a laser depositing its energy close to the fiber tip. The acoustic wave is emitted on a short time scale relative to the bubble expansion. The acoustic wave velocity for a uniform laser deposition is $u_{s}=\left(P-p_{\infty}\right) /\left(\rho c_{s}\right)$ and its energy is $e_{s}=L A \rho u_{s}^{2} / 2$, where $c_{s}$ is the adiabatic sound speed, $L$ and $A$ are the laser deposition length and area, respectively. This acoustic energy can be reduced from the bubble initial energy thus taking into account acoustic emission. At the final collapse stage of the bubble in a $2 \mathrm{D}$ case, most of the energy is dissipated and the bubble rebound is usually small.

In the following we assume that the boundary pressure, $P$, is the pressure $P_{b}$ inside the bubble. In future work several effects as surface tension, viscosity, acoustic emission, materiel strength and failure, should be included in determining the boundary pressure. ${ }^{9}$

\section{THE NUMERICAL PROCEDURE}

At the initial time $t_{0}=0$ we set the flow potential $\Phi_{0}=0$ and the velocity $U_{0}=0$ at the bubble's boundary. The initial density inside the bubble is taken as $\rho_{0}=1 \mathrm{~g} / \mathrm{cm}^{3}$ and the instantaneous laser energy deposition determines its initial temperature $T_{0}$, pressure $P_{0}$ and the constant entropy of the bubble $S_{0}$. We use $S_{0}$ to obtain the adiabat of the gas inside the bubble in a table of pressure as a function of density. An equation of state (EOS) of water is used based on NBS Steam Tables. ${ }^{10}$ Throughout the calculations we used the Unstructured Mesh Finite

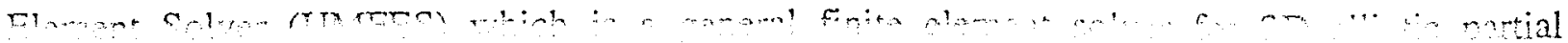


differential equations (as the Laplace equation) over an arbitrary bounded domain, with Dirichlet $(\phi=0)$, Neumann $\left((\nabla \phi)_{N}=0\right)$ or mixed boundary conditions. ${ }^{6.7}$ The density of the generated mesh is usually user-supplied, based on the nature of the given problem.

At the $n^{\text {th }}$ time step we perform the following:

Step 1: The conditions on the bubble's boundary for the potential flow are first updated by using Eq.(6),

$$
\Phi_{n}=\Phi_{n-1}+\Delta t_{n-1}\left[\frac{I}{\rho}\left(P_{n-1}-p_{\infty}\right)-\frac{U_{n-1}^{2}}{2}\right] \quad,
$$

where $P_{n-1}$ is the bubble pressure, $\rho$ the liquid density, and $\Phi_{n-1}, \dot{U}_{n-1}$ are the flow potential and the velocity at the bubble's boundary during the $(n-1)^{\text {th }}$ time step.

Step 2: We solve Laplace equation for $\phi_{n}$ outside the region using UMFES. We then update the velocities $\vec{U}_{n}$ at the bubble's boundary using the Laplace solution and the relation,

$$
\vec{U}_{n}=-\nabla \phi_{n}
$$

where the flow potentials and the velocities are calculated at the finite element mesh points.

Step 3: Update the bubble's new boundary using the velocities calculated in Step 2 and the time interval $\Delta t_{n}$.

Step 4: Update the bubble density $\rho_{n}$ by calculating the bubble's volume $V_{n}$ and using the relation,

$$
\rho_{n} V_{n}=\rho_{0} V_{0}
$$

The adiabat is used to calculate the new bubble pressure $P_{n}$. For the next step we use $\rho_{n}, P_{n}$ and $\vec{U}_{n}$ in Eq.(9).

In the numerical procedure we assume a vanishing flux on the surface of the fiber, i.e. $u_{N}=(\nabla \phi)_{N}=0$, where $N$ stands for the normal component. On the other walls we assume Dirichlet boundary conditions, i.e. $\phi=0$ provided they are far enough from the bubble.

\section{RESULTS AND DISCUSSION}

Throughout this work we assume a fiber radius $r_{f}=100 \mu m$ and a homogeneous laser absorption length of $a=7 \mu \mathrm{m}$. A typical triangulation that depends inversely on the distance from the fiber tip is shown in Fig.(1). We concentrated on two problems of different types: (a) a free boundary problem and (b) a bubble confined in a channel. 


\subsection{Case (a): A bubble expanding in a free boundary region.}

In this case the boundaries of the outside region to the fiber are far from the bubble. The boundary conditions upon them can be taken either as $\phi=0$ or $(\nabla \phi)_{N}=0$. The initial bubble is the rectangle $0 \leq z \leq 7 \mu m, 0 \leq r \leq 100 \mu m$ (see Fig.(1)). Although the boundary in Fig.(1) is shown at $300 \mu \mathrm{m}$, we used $1000 \mu \mathrm{m}$ in each direction for the following calculations.

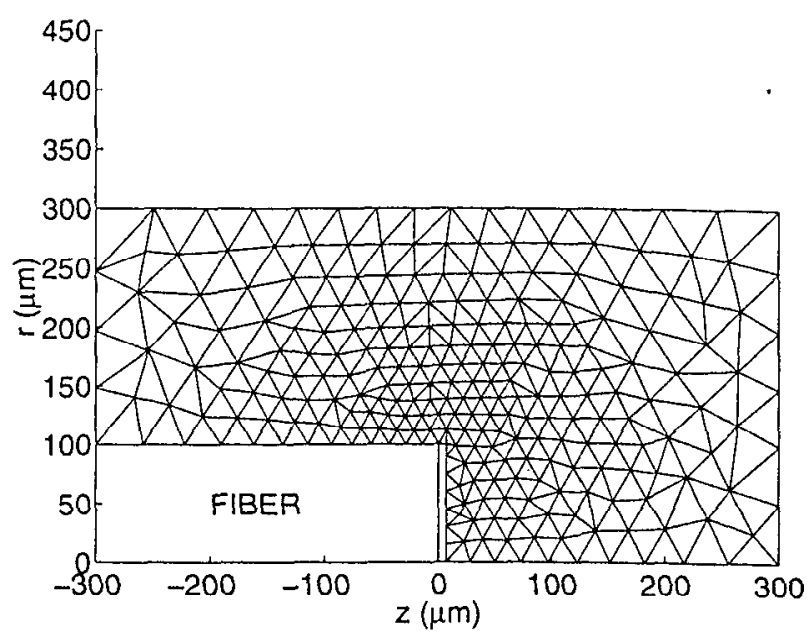

Figure 1: The initial bubble close to $\mathrm{z}=0$ and its outside region for a free boundary problem with a triangulation depending inversely on the distance from the fiber tip.

Once the system absorbs the laser energy, the bubble expands until the ambient pressure on its boundary causes it to come to rest. Then the collapse phase starts and the bubble's boundary moves in opposite direction. A typical expansion-collapse process is given in Fig.(2). In this case we assume $p_{\infty}=10 \mathrm{bar}, T_{0}=350^{\circ} \mathrm{C}$, where $T_{0}$ is the initial temperature of the bubble after the laser energy deposition. The bubble maximum expansion is at about $9 \mu s$ and its maximum radii are about $250 \mu \mathrm{m}$ to the right direction $(+\mathrm{z})$ and $200 \mu \mathrm{m}$ to the left $(-\mathrm{z})$ and in the radial ( $\mathrm{r})$ directions relative to the fiber tip corner $(z, r)=(0,100)$. The expansion and collapse to the left and radial directions occur approximately at the same time, while that to the right direction lasts longer. These causes that the bubble collapses away from the fiber [see Fig.(2) at $17 \mu \mathrm{s}$ ]. These results are consistent with experiments. ${ }^{11}$

In Fig.(2) at $0.5 \mu \mathrm{sec}$ we see a rapid growth of the bubble near the edge of the laser deposition region in the radial direction. This fact results from the solution of the Lalaplce equation that forces large velocities near corners. The behavior is analogous to the appearance of large electric fields induced at the neighborhood of charged edges. Increasing the numerical zones around the bubble boundary by a factor of four does not change the results. 

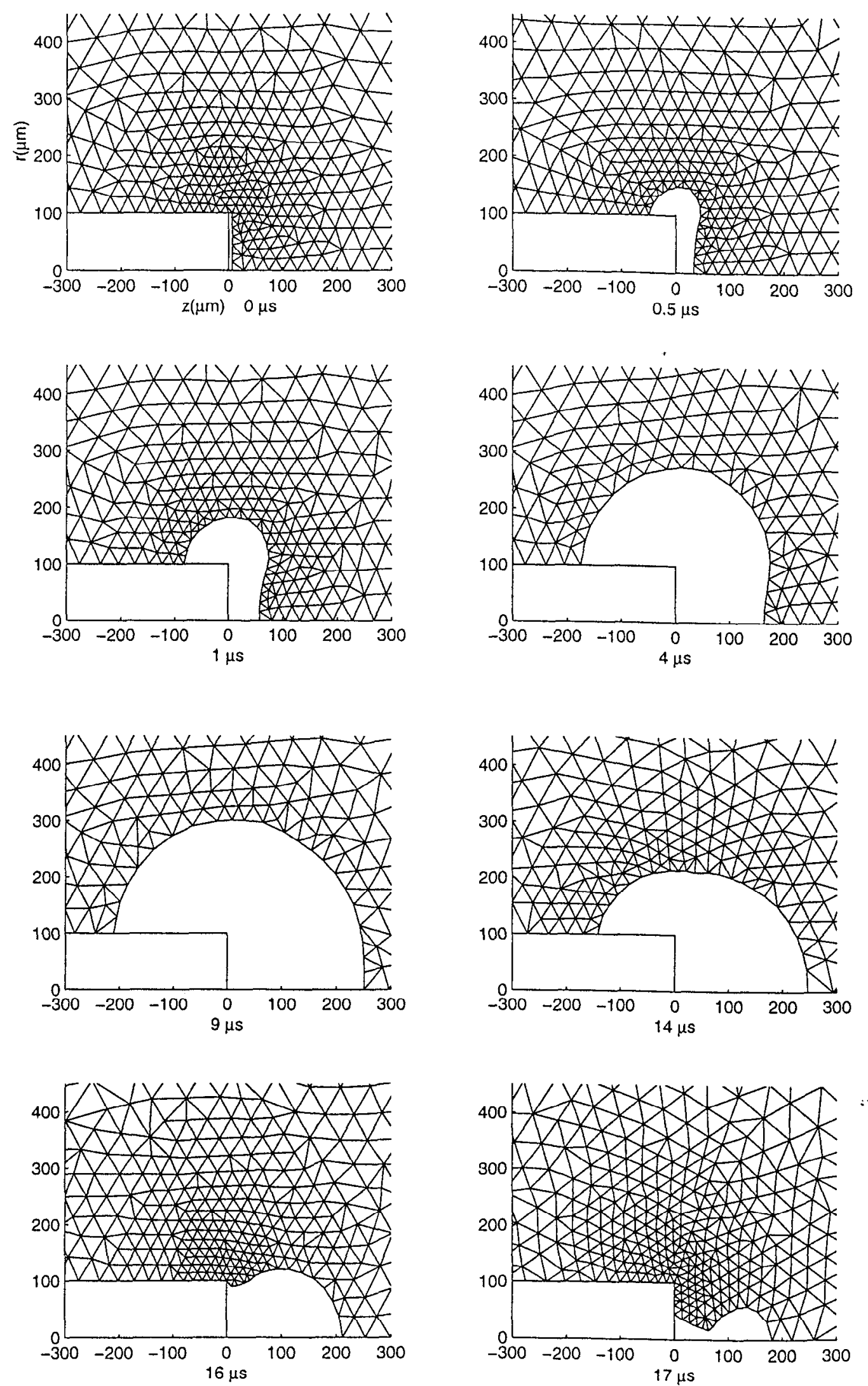

Figure 2. Bubble expansion in a free boundary: bubble expansion and collapse in cylindrical . coordinates $\mathrm{r}$ and $\mathrm{z}$ for various times, where lengths are in $\mu m$, times in $\mu s$. Here $p_{\infty}=10 \mathrm{bar}, T_{0}=350^{\circ} \mathrm{C}$. 
In Fig.(3) $r_{R}, r_{N}, r_{L}$, the bubble dimension in the right direction relative to the fiber tip edge $(0,0)$, and the bubble dimensions in radial and left directions, relative to the fiber tip edge at $(0,100)$ respectively, are given as functions of time. It can be seen that the expansion to the right lasts longer relative to the normal and left expansions. A typical relation between the maximum diminution $r_{m}$ and the time to the maximum $\tau_{m}$ for the various directions is, $r_{m} / \tau_{m}=A_{0} \sqrt{p_{\infty} / \rho}$, where $A_{0} \approx 0.7$.

From the solution to the flow potential $\phi$ we get a positive value when the bubble is expanding away from the fiber and a negative value for the collapse stage. The flow potential has a typical almost $1 /|\vec{r}|$ dependence on the distance $|\vec{r}|$ from the fiber tip in the various directions.

Fig.(4a) and (4b) represent the maximum bubble expansion in directions $r_{R}, r_{N}, r_{L}$ relative to the fiber tip edges $(0,0)$ and $(0,100)$ for constant ambient pressure $p_{\infty}$ and a varying initial bubble temperature $T_{0}$, and of a constant $T_{0}$ and a varying $p_{\infty}$. The main deviation is between the expansion to the right relative to the normal or left directions. The deviation increases with increasing $T_{0}$ and decreasing $p_{\infty}$.

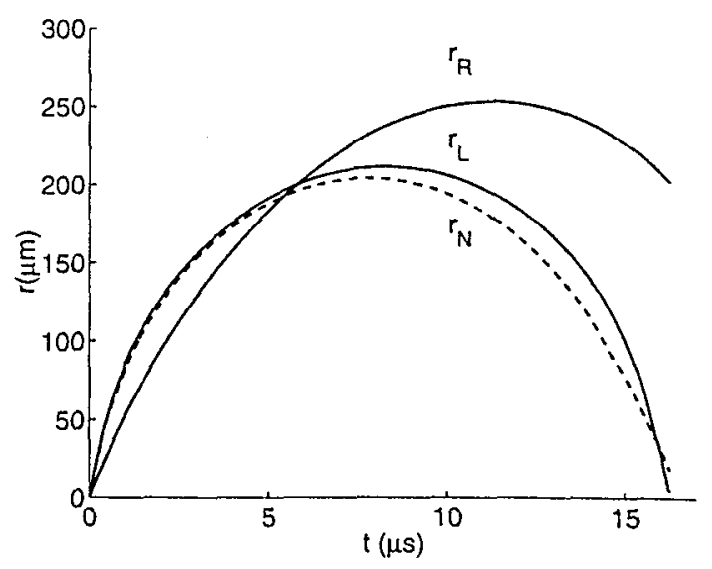

Figure 3: Free boundary: Bubble dimensions $r_{R}, r_{N}, r_{L} v s$ time for the right $\left(r_{R}, 0\right)$, normal $\left(0,100+r_{N}\right)$ and left $\left(-r_{L}, 100\right)$ directions respectively relative to the fiber tip edges. Here $p_{\infty}=10 \mathrm{bar}, T_{0}=350^{\circ} \mathrm{C}$. 

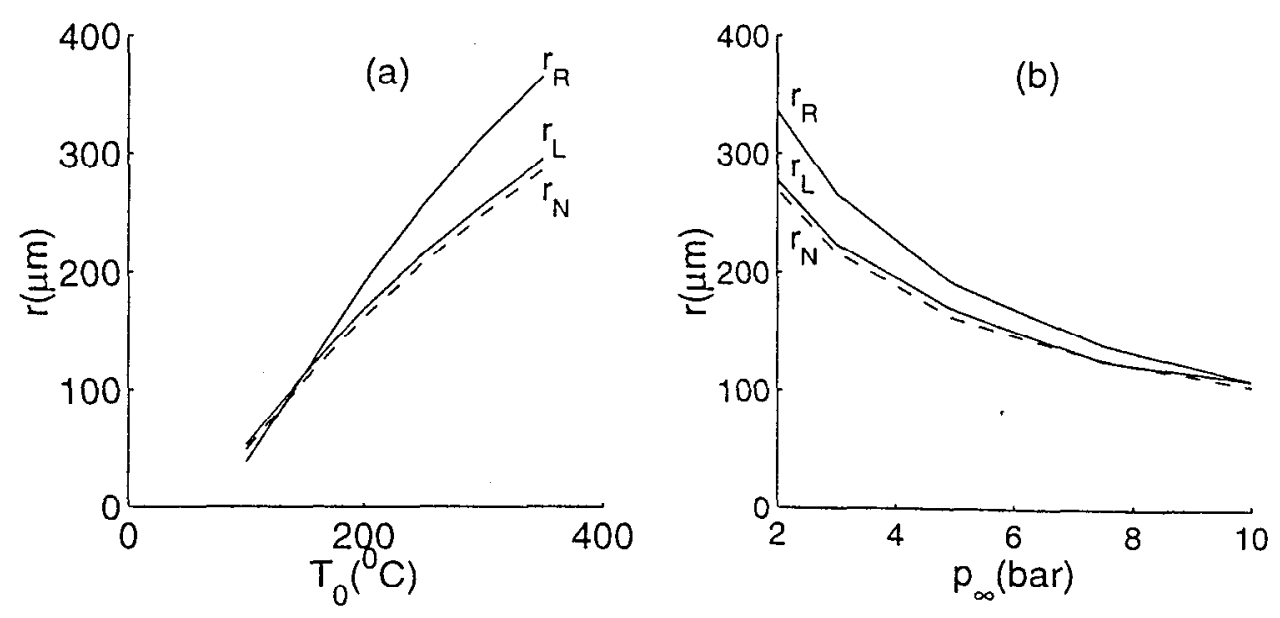

Figure 4: Bubble maximum distances in the left, normal and right directions relative to the fiber tip $\left(r_{L}, r_{N}, r_{R}\right.$ respectively), as (a) functions of $T_{0}$ for a constant $p_{\infty}=5 \mathrm{bar}$; (b) functions of $p_{\infty}$ for a constant $T_{0}=200 \mathrm{C}^{\circ}$.

In the following discussion we presents a comparison between our 2D Rayleigh model and a hydrodynamic simulation presented in Ref.(2). The system in Ref.(2) is comprised of a fiber of radius $100 \mu \mathrm{m}$ which delivers energy of $0.317 \mathrm{~mJ}$. The laser energy is deposited exponentially in the $z$ direction with an absorption length of $l_{b}$ $=7 \mu \mathrm{m}$. In the radial direction the laser deposition is uniform up to $100 \mu \mathrm{m}$ and beyond that decreases as a Gaussian with a factor of $1 / e$ in a length of $10 \mu \mathrm{m}$. The energy included in the Gaussian is $20 \%$ of the total laser energy. In our 2D Rayleigh model we include only the energy confined inside the expanding bubble. We exclude $37 \%$ of the energy outside the absorption length $l_{b}$. We also reduce $7 \%$ of the total energy included in the Gaussian tail, and $6 \%$ of the energy emitted as acoustic waves. Thus the amount of included energy, responsible for bubble expansion is about $50 \%$ of the total energy. This energy is spread homogeneously in front of the fiber tip in the $7 \mu \mathrm{m}$ absorption length. This energy imposes an initial bubble temperature of about $200^{\circ} \mathrm{C}$.

Figure (5) presents the average bubble radius defined in Ref.(2) $R_{B}=\left(a b^{2}\right)^{1 / 2}$ as a function of time, where $2 a$ is the maximum expansion of the bubble along the symmetry axis $z$ and $b$ is the maximum bubble expansion in the radial direction for a given time. In Fig.(5) the solid lines are our model results for bubble initial temperatures $180^{\circ} \mathrm{C}$ and $200^{\circ} \mathrm{C}$ and the dash line is the result of Ref.(2) in Fig.(8). A general agreement of about $120 \mu \mathrm{m}$ expansion and collapse time of about $10 \mu \mathrm{sec}$ is obtained. The maximum in $R_{B}$ is obtained earlier in Ref.(2) at $4.5 \mu \mathrm{sec}$ and in our model at about $6.5 \mu \mathrm{sec}$. We should remember that our system is just an approximation to the system solved in Ref. (2). For better agreement we should compare our model with hydrodynamic simulation for the same system. Such comparisons will be done in the future. 


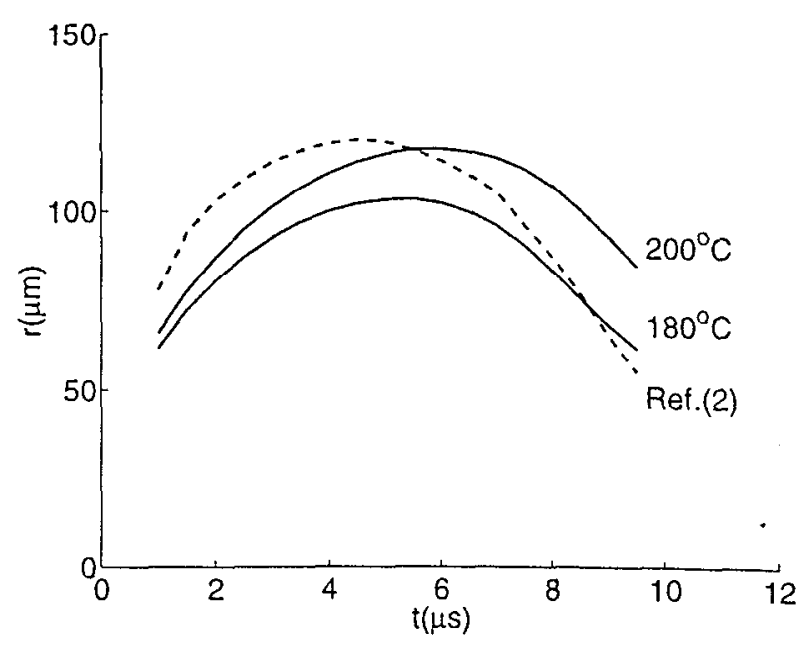

Figure 5: Bubble average radius vs time for the free boundary case, with fiber radius $100 \mu \mathrm{m}$ and $p_{\infty}=10 \mathrm{bar}$. Solid lines are our results for bubble initial temperatures $\mathrm{T}_{0}=180{ }^{\circ} \mathrm{C}$ and $200^{\circ} \mathrm{C}$. Dashed line is Ref.(2), Fig.(8) result with energy delivered of $0.317 \mathrm{~mJ}$.

\subsection{Case (b): A bubble expanding in a confined channel.}

In the case of a bubble confined in a channel, the fiber and the channel arc two coaxial cylinders with a distance $d$ between them. The boundary condition on the channel's face is homogeneous Neumann (no fluid penetration, $\left.(\nabla \phi)_{N}=0\right)$. The bubble for $p_{\infty}=10 \mathrm{bar}$, $T_{0}=350^{\circ} \mathrm{C}$ and $d=250 \mu \mathrm{m}$, at several times is shown in Fig.(6). Initially the flows are similar in the three directions to the free boundary case. Then the radial flow interacts with the channel boundary and is significantly reduced. This increases the flow mainly to the right of the channel. The expansion and collapse times are larger relative to the free boundary case. However the collapse from the right and left directions are much faster than from the radial direction, which causes a large distortion in the bubble (see Fig.(6) at $27 \mu \mathrm{s}$ ). We will consider the later time collapse in future work.

The slow expansion and collapse of the bubble in the radial direction is a consequence of the Neumann (normal component $\left.(\nabla \phi)_{N}=0\right)$ ) boundary condition on the channel. This condition presents the vessel wall elastic resistance to large deformations. While the flow potential on the bubble boundary $\phi$ in Eq.(9) depends on the ambient pressure $p_{\infty}$, the bubble boundary velocity depends on $\nabla \phi$. When the bubble expends closer to the channel wall the solution of the Laplace equation imposes a flat flow potential in the radial direction connecting the bubble boundary and the channel wall. The flat potential causes slow expansion and collapse in the radial direction. This is related to the incompressibility of the fluid, imposing higher flows in both the $+z$ and $-z$ directions. In contrast by selecting the Dirichlet boundary condition $(\phi=0)$ on the channel boundary, we obtain significant increase of the expansion and collapse of the bubble in the radial direction.

An important property, which we ignore here, is the acoustic wave emission by the bubble. These waves can be partially reflected by the channel wall and affect the bubble expansion. The effect was considered in Ref. (2) by hydrodynamic simulation using an 
appropriate EOS for the channel wall. This effect further delays the bubble expansion and collapse. However, in Ref.(2) the channel wall resistance to large deformations was ignored.
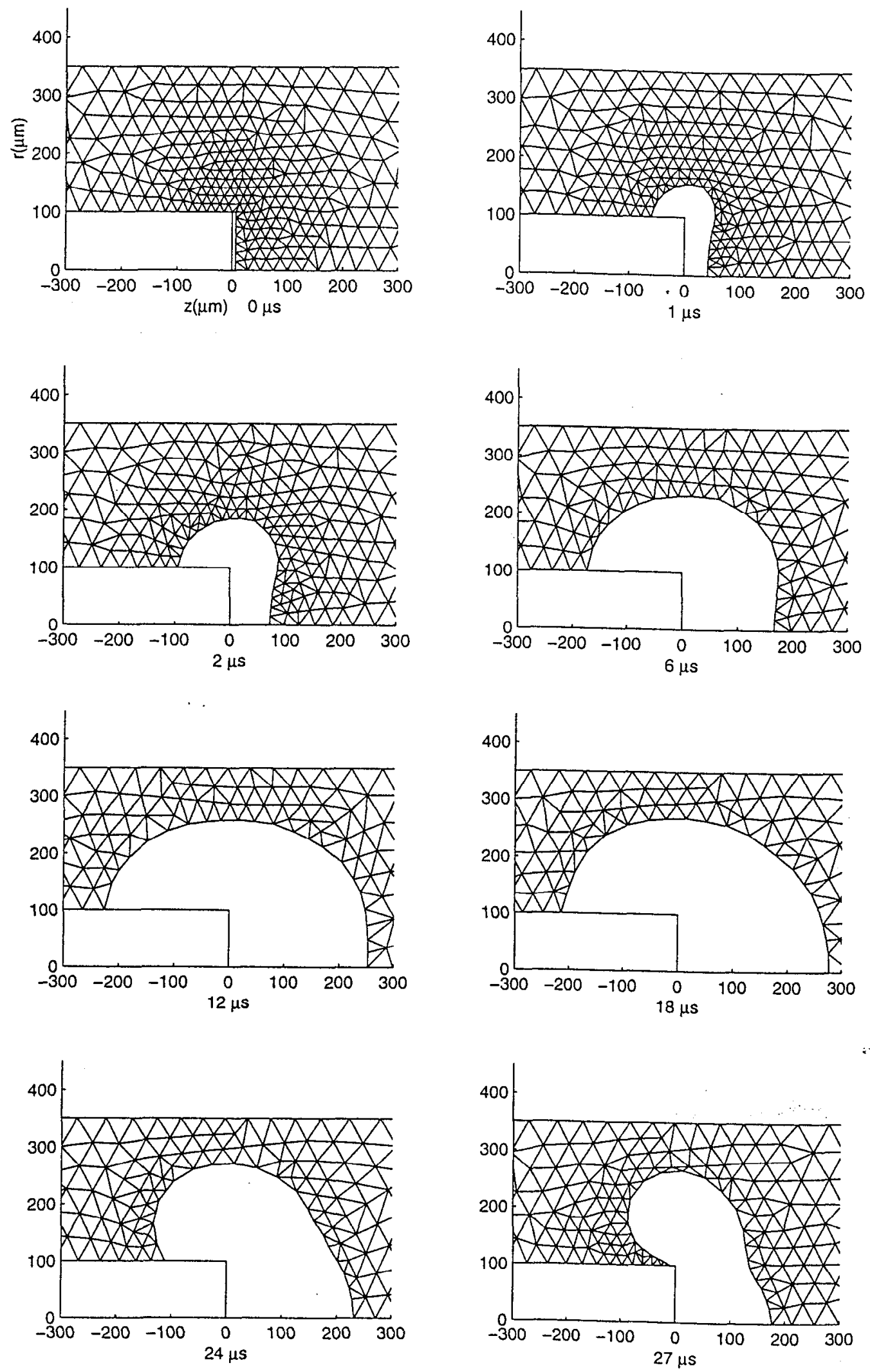
Figure 6: Bubble confined in a channel: expansion and collapse in cylindrical coordinates $r$ and $z$ for various times where lengths are in $\mu \mathrm{m}$ and times in $\mu \mathrm{s}$. Here $p_{\infty}=10 \mathrm{bar}, T_{0}=350^{\circ} \mathrm{C}$.

The three distances $r_{R}, r_{N}, r_{L}$ in the right, radial and left directions as functions of time are plotted in Fig.(7). Initially, expansion is similar in the three directions till the radial flow approaches the channel boundary and the radial distance is reduced relative to the others. The flow potentials from the bubble's edges to the right and left directions are a decreasing function of the distance. The radial direction has a flat flow potential and consequently a very slow flow relative to the right and left directions.

In Figs.(8a), (8b) we present the expansion time $z$ needed for full expansion and the maximum distance $r$ in all three directions as functions of the channel dimension $d$. This gives the asymptotic behavior which connects channeled systems and a free boundary system as $d$ increases to large values. The expansion and collapse periods in radial direction are much longer in the channeled case than in the case of free boundaries. This causes the collapse from the right and from the left, to progress faster than from the other directions and consequently, a completely different structure of a collapsed bubble is obtained.

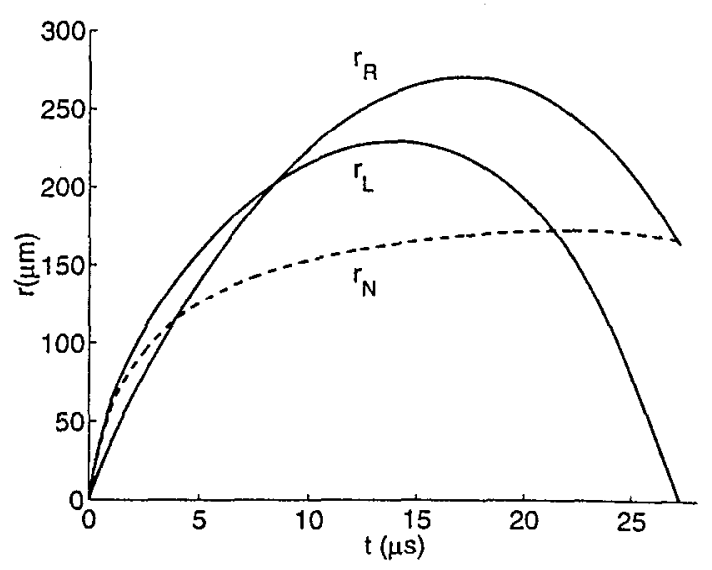

Figure 7: Bubble confined in a channel: bubble dimensions $v s$ time for the right, normal and left directions relative to the fiber tip edges, $r_{R}, r_{N}, r_{L}$, respectively. Here $p_{\infty}=10 \mathrm{bar}$ and $T_{0}=350^{\circ} \mathrm{C}$. 

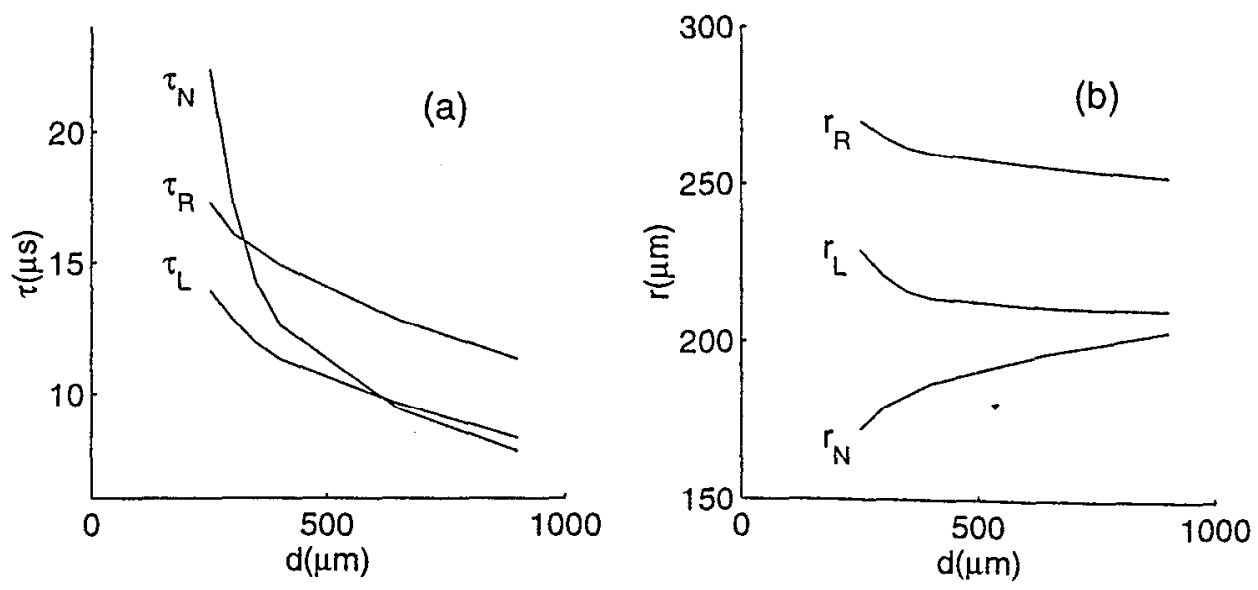

Figure 8: (a) Time $\imath$ and (b) distance $\mathrm{r}$ for maximum expansion, as functions of $d$, for right, normal and left directions. Here $p_{\infty}=10 \mathrm{bar}, T_{0}=350^{\circ} \mathrm{C}$.

\section{CONCLUSIONS}

A potential flow method was applied to obtain a 2D Rayleigh type model for bubble expansion and collapse. The main assumption is that the inside of the bubble is homogeneous in pressure and density as is usual assumed in a Rayleigh model. The method is based on solving a Laplace equation for the flow potential in the outer bubble region based on a moving boundary condition for the bubble boundary. The 1D Rayleigh bubble expansion is a special case of our 2D model. We find the method flexible to consider various tissue boundary conditions and geometry. We solve a fiber in a free boundary as well as a fiber in a channel. The method can be applied in various realistic medical applications.

The 2D Rayleigh model presented here is much faster and simpler than the 2D compressible hydrodynamic simulation. It can be applied for design and understanding of fiber based medical therapies. The accuracy of the method should be tested by detail comparison with hydrodynamic simulations.

This flow potential method should be extended to include other physical mechanisms that affect tissue behavior as: surface tension, viscosity, acoustic emission, and strength and failure properties.

\section{ACKNOWLEDGMENT}

The work of R. London and P. Amendt was preformed under the auspices of the U.S. Department of Energy by the Lawrence Livermore National Laboratory under Contract No. W7405-Eng-48. 


\section{REFERENCES}

1. K.W. Gregory, "Laser thrombolysis", Interventional Cardiology (E.J. Topol, editor, W.B. Sanders \& Co.), 2 (1994) p. 892.

U. Sathyam, A. Shearin, and S. Prahl, Lasers in Surgery VI 2671, 28 (1996).

M. Strauss, P. Amendt, R. A. London, D. J. Maitland, M. E. Glinsky, P. Celliers, D. S. Baily and D. A. Young, "Computational modeling of laser thrombolysis for stroke treatment", in Laser in Surgery VI, Proc. SPIE 2671 (1996), p.11. 1st.Vogel, S. Busch and U. Parlitz, "Shock wave emission and cavitation bubble generation by picosecond and nanosecond optical breakdown in water", J. Acoust. Soc. Am., 100 (1996) p. 148.

2. P. Amendt, M. Strauss, R. A.London, M. E. Glinsky, D. J. Maitland, P.M. Celliers, S.R. Visuri, D. S. Baily, D. A. Young and D. Ho, “ Modeling of bủbble dynamics in relation to medical applications", Proceedings of Laser Tissue Interaction VIII, SPIE 2975, (1997) P. 362.

3. Lord Rayleigh, "On the pressure developed in a liquid on the collapse of a spherical cavity", Philos. Mag. 34, (1917) p. 94. M. Plesset, "The dynamics of cavitation bubbles", J. Appl. Mech. 16, 277 (1949).

4. R.T. Knapp, J. W. Daily and F.G. Hammitt, Cavitation (McGraw Hill, New York), p. 94.

5. L.D. Landau and E.M. Lifshitz, Fluid Mechanics (Pergamon Press 1987) p. 14.

6. M. Friedman and A. Kandel, "On the design of fuzzy intelligent differential equation solver", in Fuzzy Expert Systems, A. Kandel, Ed., CRC Press, Boca Raton,1994.

7. M. Friedman and A. Kandel, Fundamentals of computer Numerical Analysis, CRC Press, Boca Raton, 1994.

8. E.J. Chapyak and R.P.Godwin, "Comparison of numerical simulations and laboratory studies of shock waves and cavitation bubble growth", Proceedings of Laser Tissue Interaction VI, SPIE 2975, (1997) p. 335.

9. M.E. Glinsky, P. A. Amendt, D. S. Bailey, R. A. London, A. M. Rubenchik and M. Strauss, "Extended Rayleigh model of bubble evolution with material strength compared dynamic simulations"', Proceedings of Laser Tissue Interaction VI SPIE 2975, (1997) p. 318.

10. L. Haar, J.S. Gallagher and G.S. Kell, NBS/NRC Steam Tables (McGraw-Hill, New York, 1984).

11. P. Celliers, L. Da Silva, N.J. Heredia, B. M. Mammini, R.A. London and M. Strauss, "Dynamic of laser-induced transients produced by nanosecond duration pulses", in Laser in Surgery VI, Proc. SPIE 2671 (1996), p. 11. 